Louveau, Frédérique. Un prophétisme japonais en Afrique de l'Ouest. Anthropologie religieuse de Sukyo Mahikari (Bénin, Côte-d'Ivoire, Sénégal, France)

Paris, Karthala, 2012

\title{
Anne Mélice
}

\section{OpenEdition}

\section{Journals}

Édition électronique

URL : http://journals.openedition.org/etudesafricaines/18162

DOI : 10.4000/etudesafricaines. 18162

ISSN : $1777-5353$

Éditeur

Éditions de l'EHESS

Édition imprimée

Date de publication : 6 juillet 2015

ISSN : 0008-0055

\section{Référence électronique}

Anne Mélice, «Louveau, Frédérique. Un prophétisme japonais en Afrique de l'Ouest. Anthropologie religieuse de Sukyo Mahikari (Bénin, Côte-d'Ivoire, Sénégal, France) », Cahiers d'études africaines [En ligne], 218 | 2015, mis en ligne le 01 janvier 2015, consulté le 24 septembre 2020. URL : http://

journals.openedition.org/etudesafricaines/18162; DOI : https://doi.org/10.4000/etudesafricaines. 18162

Ce document a été généré automatiquement le 24 septembre 2020.

(C) Cahiers d'Études africaines 


\section{Louveau, Frédérique. Un prophétisme japonais en Afrique de l'Ouest. Anthropologie religieuse de Sukyo Mahikari (Bénin, Côte-d'Ivoire, Sénégal, France)}

Paris, Karthala, 2012

Anne Mélice

LOUVEAU, Frédérique. - Un prophétisme japonais en Afrique de l'Ouest. Anthropologie religieuse de Sukyo Mahikari (Bénin, Côte-d'Ivoire, Sénégal, France). Préface de Georges Balandier. Postface de Jean-Pierre Dozon. Paris, Karthala, 2012, 502 p., bibl., gloss., ill.

1 Le livre de Frédérique Louveau, issu de sa thèse de doctorat, est consacré à l'implantation en Afrique d'un prophétisme japonais, Sukyo Mahikari. Fondé en 1959 au Japon, ce mouvement n'est pas conçu, du point de vue émique, comme une religion, mais est appelé " groupe spirituel » (ou encore " art spirituel », « art initiatique » ou " art de vivre ») par ses membres, lesquels, eux-mêmes, se désignent comme des «initiés ». Au cours des années 1960, il gagna la France, puis l'Afrique au cours des années 1970. L'étude présente, en regard des travaux précédemment consacrés à Sukyo Mahikari, la spécificité de se déplacer du contexte japonais vers d'autres lieux de son implantation, en France et en Afrique, et de faire ainsi droit à sa portée globalisante. Rien d'arbitraire dans le choix de la France et de l'Afrique : parmi les cinq « délégations régionales » qui structurent les implantations de Sukyo Mahikari sur la planète, l'Europe et l'Afrique sont en effet regroupées au sein d'une même délégation : «La 
quasi-simultanéité dans les dates d'implantation de cette "nouvelle religion" entre la France et l'Afrique laisse penser qu'elle voyagea dans une zone franco-africaine : de la Côte-d'Ivoire, elle s'implanta au Sénégal, au Bénin puis dans les anciennes colonies belges (Congo et Rwanda) et portugaises (Angola)» (pp.152-153). Il n'est possible ici que de laisser pressentir le considérable travail de terrain dont cette thèse se fait longuement l'expression. La méthode comparative soutient une recherche ambitieuse, visant à dégager « variants » et « invariants » entre les implantations étudiées.

2 L'anthropologue opère une confrontation à quatre termes, inspirée - «très librement " à certains égards, note-t-elle - de l'idée et des techniques d'enquête multisituée de George Marcus. Partie d'une enquête ethnographique menée dans le lieu de culte, le dojo situé à Aix-en-Provence, la recherche s'est développée dans une perspective résolument comparatiste, à l'intersection du local et du global. Le branchement (au sens conceptuel explicitement emprunté à Jean-Loup Amselle) des initiés au réseau mondial trace un fil fort à travers l'ouvrage. Les dernières pages montrent d'ailleurs que, dans les représentations des initiés, le siège mondial de l'Organisation, Suza, s'offre comme le haut-lieu spirituel enchanté, le lieu des pèlerinages, celui où «s'entrecroisent la localité, c'est-à-dire la source du divin aux yeux des initiés, et la globalisation du religieux puisque les adeptes du monde entier s'y retrouvent ; c'est dans cet entrecroisement localisé que la globalité entre dans les corps des individus » (p. 472).

3 L'essor du mouvement s'éclaire de l'occidentalisation de l'Extrême-Orient et notamment de l'ouverture de son fondateur à l'Occident et au christianisme. Pareille ouverture a facilité l'initiation des adeptes occidentaux et africains. D'abord situé durant les années 1960 à Paris, où il suscita l'adhésion de Français séduits par la dimension spirituelle qui s'associe aux techniques du corps, activé par un ressourcement au Japon, le mouvement atteignit la Côte-d'Ivoire et s'y développa de manière contingente, puisque résultant de la décision prise par une initiée française d'y suivre son mari, avant de s'étendre aux autres pays d'Afrique de l'Ouest.

Cet essor ne résulte pas d'un processus diasporique. Les groupes locaux sont formés de gens du pays. Or, les différences sont considérables, non seulement entre la France et les trois pays africains, mais aussi entre ces trois pays eux-mêmes. L'islam constitue la religion dominante du Sénégal ; la France, de tradition catholique, se caractérise par sa gestion de la laïcité ; la Côte-d'Ivoire, qui compte le plus grand nombre d'adeptes de Sukyo Mahikari en Afrique, est aussi l'espace d'un déploiement important de «nouveaux mouvements religieux»; le Bénin voit les religions dites traditionnelles continuer de vivre à côté de la religion catholique et d'un islam qui se concurrencent.

5 Cependant, l'observation participante révèle une forte homogénéisation de l'espace de culte au sein duquel les rituels s'accomplissent de manière similaire : le rituel okiyome, « clé de voûte de Sukyo Mahikari » (p. 184), se déroule de la même façon partout dans le monde, dans tous les dojos. En outre, une même "vision apocalyptique du monde » (p.418) supporte chez les initiés la construction d'une nouvelle civilisation. «Les enseignements sont largement empreints de shinto et le rituel principal (okiyome) consiste en la transmission, par des initiés, de la Lumière du dieu Su à travers la paume de la main à des points précis du corps. Destiné à se purifier des impuretés spirituelles contenues dans les corps, les âmes et dans l'environnement, ce rituel implique une élévation spirituelle et une restauration du monde pour l'établissement du paradis sur la terre et d'une nouvelle "Civilisation", celle de Yoko. Les activités spirituelles se 
traduisent par des enjeux de guérison et des actions écologistes dans les différentes sociétés où le mouvement est implanté » (pp. 15-16).

6 Si les initiés sont surtout d'origine chrétienne, quelquefois musulmane, il est remarquable que soit encouragée leur double appartenance religieuse. Cette dernière commande à l'élaboration par les initiés de "petits arrangements symboliques avec leurs propres convictions religieuses » (p. 243). F. Louveau s'appuie sur la réactivation du concept lévi-straussien de «bricolage» pour comprendre le travail qu'ils accomplissent à partir du «marquage culturel japonais du matériel symbolique emprunté ", de ces " précontraintes » que constituent notamment les mots en japonais dans la liturgie, ou encore l'habillement et les rapports aux objets sacrés. Elle discerne finement la singularité des formes que prennent dans l'adhésion à Sukyo Mahikari les "juxtapositions de contradictoires" qu'André Mary avait dégagées dans le culte d'Eboga: les logiques qui distinguent les initiés sont des effets de leur religion d'appartenance, non de leur nationalité.

7 L'un des traits saillants de cette étude réside dans la mise en évidence d'un prophétisme spécifique. L'auteure montre que plusieurs éléments rapprochent la figure du leader japonais et celle de prophètes africains : « La survie miraculeuse à la mort, une retraite énigmatique, un séjour hors du monde au moment de la révélation d'un message de la part d'une divinité, une réinterprétation de la naissance et de la vie du personnage à l'aune de cette nouvelle nature divine en faveur d'un destin extraordinaire» (pp. 473-474). Toutefois, si la réception africaine fut favorisée par le rôle des prophétismes, particulièrement foisonnants en Côte-d'Ivoire, et si les convergences ne manquent pas, qui autorisent ces greffes, il reste qu'à divers égards la figure du fondateur, Okada Yoshikazu, rebaptisé Sukuinushisama lors de la fondation de sa "nouvelle religion ", diffère de celles des prophètes africains. Né en 1901, Okada eut d'abord une carrière militaire. Une maladie l'amena à quitter l'armée en 1941, et une guérison jugée miraculeuse entraîna sa transformation existentielle. C'est d'un véritable renversement axiologique que se réclame son discours prophétique, porteur d'un projet de paix et de bien-être. Ainsi aurait-il déclaré «incarner le repentir de Nobunaga »: significativement, l'hagiographie affirme qu'il est le descendant de ce dictateur du XVI ${ }^{\mathrm{e}}$ siècle, déjà ouvert, lui aussi, à l'Europe et au christianisme.

C'est dire que ce prophétisme ne plonge pas dans les couches les plus pauvres, mais présente une origine aristocratique dont le mouvement restera marqué, puisqu'il sera moins occupé d'expansion que d'élitisme, d'un souci de « distinction ", au sens imprimé par Pierre Bourdieu à ce terme, qui va de pair avec une dimension volontairement minoritaire. À cette disposition collective obéit, par exemple, l'implantation, au sein de la géographie urbaine, des lieux sacrés « dans les quartiers les moins populaires, pour ne pas dire les plus distingués» (p. 177). La sociologie des adeptes fait apparaître la particularité de Sukyo Mahikari dans le paysage contemporain des «nouveaux mouvements religieux »: les initiés sont des fonctionnaires et des nationaux, vivant dans les villes (Sukyo Mahikari est surtout implanté dans les capitales), travaillant dans l'enseignement, les ministères et le monde hospitalier. Ils sont jeunes et instruits. Ces populations favorisées mais atteintes, à la fin des années 1990, par la vulnérabilité de l'État, se voyaient proposer les services d'un perfectionnement de soi, d'une purification de soi-même en même temps que de l'environnement, dont l'auteure dégage la portée tout à la fois herméneutique et thérapeutique en usant du syntagme de « souci de soi », remis en lumière par Michel Foucault : «D'un côté, le "souci de soi" 
élaboré par les initiés est orienté vers le travail de purification des corps qui les conduit à veiller au seuil entre l'intérieur et l'extérieur [...], notamment à travers un soin apporté à leur alimentation dans un souci de ne pas ingurgiter la souillure. De l'autre côté, le souci de soi permet aux initiés de gérer la toxicité du rapport aux autres » (p. 360). Les pages consacrées aux discours d'initiés de Côte-d'Ivoire et du Bénin relatifs à la sorcellerie dont ils se disent menacés et à la protection que le travail de purification peut receler, sont des plus suggestives, qui montrent comment Mahikari leur permet de se maintenir à " un niveau spirituel élevé », soustrait - selon les termes mêmes d'un entretien (p. 368) - à l'espace d'« en bas » où les sorciers les attendent.

L'analyse complexifie la relation entre les «nouvelles religions » et la crise. En effet, l'essor de Mahikari apparaît lié au développement économique et à la croissance. Son implantation supposait des «conditions socio-économico-politiques» (p.116) qui constituent autant de traits globalement partagés, et qui le différencient des Églises prophétiques : ainsi de l'existence d'États forts et interventionnistes, de chefs d'États charismatiques (le Général de Gaulle, Félix Houphouët-Boigny, Léopold Sédar Senghor, Mathieu Kérékou) et d'un contexte de croissance économique qui contredit l'explication de l'implantation de "nouveaux mouvements religieux» par «le paradigme de la crise» (p.122). Subtilement, l'auteure fait apparaître que l'implantation de Sukyo Mahikari en une période faste entretient certes quelque rapport, mais différé, avec la crise. Reprenant l'expression que Jean-Pierre Dozon appliqua au prophète ivoirien Gbahié Koudou Jeannot, elle soulève la question de savoir dans quelle mesure Sukyo Mahikari peut faire figure d'" annonciateur de la crise " (p. 125).

10 Dans sa préface intitulée "Les religiosités et l'État ", Georges Balandier - dont l'anthropologie politique constitue une référence déclarée - met en évidence la singularité de ce mouvement qui, à la différence des mouvements en situation coloniale, ne conteste pas l'État, mais se dresse contre ses carences et ses perversions. F. Louveau constate (p. 404) que bien plutôt qu'ils ne sont des contestataires de l'État, les initiés sont des attestataires qui réclament davantage d'État en faveur d'une gestion meilleure de la société. Si le mouvement revendique son apolitisme, il serait faux d'ignorer que les adeptes s'emploient à agir dans le monde. À côté de l'engagement politique de certains initiés africains, ils sont nombreux à témoigner du souci de l'environnement et à pratiquer des activités ressortissant à l'agriculture de subsistance et des activités écologiques. Au Sénégal, par exemple, la "vision apocalyptique " commande à la formation des jeunes «à la protection des rapports sociaux et à la restauration du monde» (p. 426). Plutôt qu'ils ne se désolent des injustices passées, c'est vers l'avenir que se tournent résolument les initiés de Mahikari. Pour résumer lapidairement cette vision du monde, F. Louveau infléchit vers l'action écologique la fameuse prescription héritée de Marx : il s'agirait en somme de « changer le monde par une gestion de l'environnement » (p. 426).

11 Tant G. Balandier, dans sa préface, que J.-P. Dozon, dans sa postface, soulignent l'apport de cette recherche : en écrivant, le premier, que son auteure « a innové en étudiant sur le terrain la rencontre de deux univers syncrétiques, l'un asiatique, l'autre africain »; le second, qu'en se déplaçant dans l'espace franco-africain, elle aboutit à des résultats "qu'aucune autre enquête n'aurait pu mettre en évidence». C'est qu'en effet, son originalité est indissociable de la solidité de l'enquête de terrain qui la supporte. 